\title{
One tamed at a time: A new approach for controlling continuous magnitudes in numerical comparison tasks
}

\author{
Moti Salti $^{1,2}$ • Naama Katzin ${ }^{1,3}$ - David Katzin ${ }^{4}$ - Tali Leibovich ${ }^{2,5}$ - Avishai Henik ${ }^{1,3}$
}

Published online: 20 July 2016

(C) Psychonomic Society, Inc. 2016

\begin{abstract}
Non-symbolic stimuli (i.e., dot arrays) are commonly used to study numerical cognition. However, in addition to numerosity, non-symbolic stimuli entail continuous magnitudes (e.g., total surface area, convex-hull, etc.) that correlate with numerosity. Several methods for controlling for continuous magnitudes have been suggested, all with the same underlying rationale: disassociating numerosity from continuous magnitudes. However, the different continuous magnitudes do not fully correlate; therefore, it is impossible to disassociate them completely from numerosity. Moreover, relying on a specific continuous magnitude in order to create this disassociation may end up in increasing or decreasing numerosity saliency, pushing subjects to rely on it more or less, respectively. Here, we put forward a taxonomy depicting the relations between the different continuous magnitudes. We use this taxonomy to introduce a new method with a complimentary Matlab toolbox
\end{abstract}

Moti Salti and Naama Katzin contributed equally to this article

Electronic supplementary material The online version of this article (doi:10.3758/s13428-016-0772-7) contains supplementary material, which is available to authorized users.

Moti Salti

motisalti@gmail.com

1 Zlotowski Center for Neuroscience, Ben-Gurion University of the Negev, Beer-Sheva, Israel

2 Brain Imaging Research Center, Ben-Gurion University of the Negev, Beer-Sheva, Israel

3 Department of Psychology, Ben-Gurion University of the Negev, Building 95, P.O.B. 653, Beer Sheva 84105, Israel

4 School of Mathematical Sciences, Tel-Aviv University, Tel-Aviv, Israel

5 Numerical Cognition Laboratory, The University of Western Ontario, Toronto, Ontario, Canada that allows disassociating numerosity from continuous magnitudes and equating the ratio of the continuous magnitudes to the ratio of the numerosity in order to balance the saliency of numerosity and continuous magnitudes. A dot array comparison experiment in the subitizing range showed the utility of this method. Equating different continuous magnitudes yielded different results. Importantly, equating the convex hull ratio to the numerical ratio resulted in similar interference of numerical and continuous magnitudes.

Keywords Non-symbolic number · Ratio effect · Matlab toolbox

\section{Introduction}

The interplay of continuous magnitudes and numerosity in size comparison tasks is controversial. Mainly, various ways for controlling continuous magnitudes have led to conflicting results (Smets, Sasanguie, Szücs, \& Reynvoet, 2015). In the current paper, we introduce a new method that enables examination of each continuous magnitude separately and minimizes saliency differences.

\section{The inseparability of numerosity and continuous magnitudes}

In the field of numerical cognition, the dominating theories postulate that humans and animals have an innate ability to perceive and manipulate numerosities, that is, a "number sense" (Cantlon, Platt, \& Brannon, 2009; Dehaene, 1997; Feigenson, Dehaene, \& Spelke, 2004). A prevalent method for studying the "number sense" is to use non-symbolic stimuli, typically with arrays of dots. The advantages of non-symbolic stimuli are manifold: They can be used on populations that are not familiar with 
numerals, like young children, infants, and animals, and they are not culture dependent.

However, in addition to numerosity, non-symbolic stimuli entail continuous magnitudes (e.g., total surface area, density, etc.) that correlate with numerosity. For example, when all dots are presented in the same size (e.g., Chassy \& Grodd, 2012) and participants are asked to decide which group contains more dots, ten dots have twice as much total surface area as five dots. In such tasks, it is impossible to know if participants responded according to the number of dots, the total area of the dots, or used a combination of both area and numerosity.

\section{Methods of controlling for continuous magnitudes in numerical comparison tasks}

The possible confounds of continuous magnitudes in numerosity judgment experiments were recognized even by those who supported the notion of a "number sense." Piazza and colleagues (Piazza, Izard, Pinel, Le Bihan, \& Dehaene, 2004) tried to dissociate continuous magnitudes from numerosity by eliminating the correlation between total surface area and numerosity, thus making total surface area an uninformative cue of numerosity. Piazza et al. suggested the results obtained under these controlled conditions should be attributed to the processing of numerosity and not to continuous magnitudes. However, this method was criticized on several grounds (Gebuis \& Reynvoet, 2011; Leibovich \& Henik, 2013, 2014; Leibovich, Henik, \& Salti, 2015). Mainly, disassociating a single continuous magnitude ignores the possibility that participants might rely on several continuous magnitudes or even switch between them (see Leibovich \& Henik, 2014).

Gebuis and Reynvoet $(2011,2012 b)$ created a code that manipulates congruency between numerosity and one or several continuous magnitudes. In congruent trials, the more numerous array was larger on these continuous magnitudes. In incongruent trials, the less numerous array was larger on these continuous magnitudes. In addition, their code recorded all continuous magnitudes to enable statistical monitoring of their distances. Analysis of Gebuis and Reynvoet's stimuli showed that the numerical and continuous magnitudes and their distances did not correlate. In numerical cognition studies, it was well established that it is easier to differentiate between two numerosities as their numerical distance increases (i.e., the distance effect; Buckley \& Gillman, 1974; Moyer \& Landauer, 1967).

Recent studies that used this method demonstrated that continuous magnitudes still affect participants' performance in numerical decisions. Leibovich and Henik (2014) presented participants with pairs of dot arrays containing 5-25 dots each and asked them to choose the group containing more dots. They found that the ratio between the continuous magnitudes accounted for half of the explained variance in response times. In an electroencephalogram (EEG) study, Gebuis and Reynvoet
(2013) used repetitive presentation of the relevant magnitudes but changed the other magnitudes (i.e., habituation paradigm) and measured separately habituation to numerosity and to continuous magnitudes. They did not find a brain activity associated with dishabituation to numerosity but did find one associated with dishabituation to continuous magnitudes at lateral occipital and parietal electrodes. Such findings challenge the notion that in the context of numerical judgments, number is the only visual property that is extracted from non-symbolic presentation of quantities. However, the contribution of continuous magnitudes should be examined meticulously.

\section{Caveats in the existing methods}

Gebuis and Reynvoet's (2011, 2012a) attempt to monitor the distance of continuous magnitudes suffers from some potential caveats. The measurement units of continuous magnitudes are arbitrary (e.g., pixels, centimeters, inches, etc.). The units of numerosity, on the other hand, are discrete and constant (dots, in the case of dot arrays). This makes the distance between continuous magnitudes uninformative and unscaled. The lack of suitable and generalized units prevents compatibility of the numerical and continuous values and distances. This incompatibility could, in turn, lead to unequal saliency of the numerical differences and the continuous magnitude differences. To illustrate, consider the case where one chooses arbitrary units and creates a dot array that contains five and ten dots and, respectively, a continuous measure of 10 and 15 . Although the numerical and continuous distances are both 5 , the numerical ratio is 0.5 and the continuous ratio is 0.67 . According to Weber's law (Shepard, Kilpatric, \& Cunningham, 1975), their subjective impact would be different (i.e., the numerical difference would be more salient).

The saliency problem is multifaceted. First, the saliency of the congruency conditions can vary and affect participants' subjective correlation. To illustrate, imagine one possible extreme case in which the differences in the congruent condition are distributed normally around a mean, and the differences in the incongruent condition are distributed around the same mean but in a Ushape distribution. In this case, although the differences would not correlate with numerosity, the same threshold would leave out more cases in the U-shaped distribution. This might result in a subjective distribution that favors the normally distributed condition, in this example the congruent condition (in contrast to the objective distribution of equal congruent and incongruent trials; see Fig. 1). In other words, if the differences in congruent and incongruent trials do not share the same distribution, the differences of one condition could be more salient, creating an alternative explanation to the congruity effect (i.e., the differences between congruent and incongruent trials can occur not because of automatic processing of the irrelevant dimension but because of different saliency between the two conditions). Second, differences in saliency between numerosity and continuous 


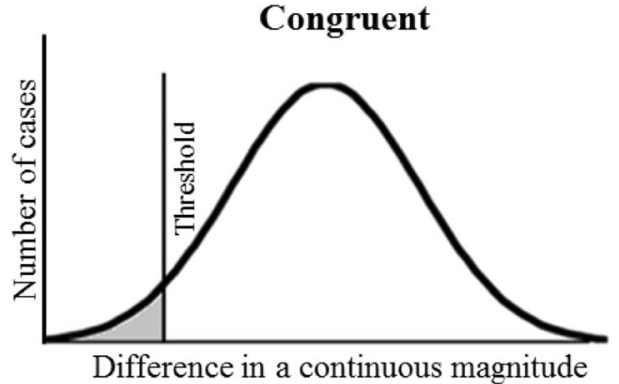

Fig. 1 Example of how the congruency conditions can differ in saliency. In this example, the congruent condition is normally distributed and the incongruent condition has a U-shape distribution. For a similar threshold
Incongruent

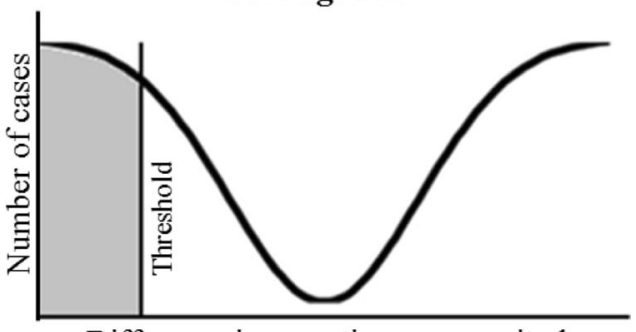

Difference in a continuous magnitude

between the two conditions, more cases would be left out of the incongruent condition

manipulated separately from numerosity, they are inseparable from each other. To this end, we put forward a taxonomy that would shed light on the mathematical properties and relations of the continuous magnitudes.

The continuous magnitudes can be divided into two groups (Fig. 2): The intrinsic features group and the extrinsic feature group. The intrinsic group describes features that can be calculated for each individual dot, such as the diameter, its circumference, and area. The intrinsic group has two sub-types of magnitudes: The first sub-type includes magnitudes in which the radii are to the first power (i.e., have an exponent of 1): total circumference and average diameter (they are a linear transformation of one another; Formulas 1-2). The second sub-type includes magnitudes in which the radii are to the second power (i.e., have an exponent of 2): total surface area (Formula 3). The second group describes the extrinsic features, that is, features that can only be calculated on an array of dots. These extrinsic features take into account not only the size of each item (radius square) but also their location. This group of features includes the area of the convex hull and density, which are negatively correlated with each other (Formulas 4-5).

These different mathematical characteristics of each group have methodological implications. They demonstrate that when numerosities are not equal, it is impossible to equate the ratio of all the continuous magnitudes to the ratio of the numerosity. To illustrate, we define numerosity ratio as $N=\frac{N_{1}}{N_{2}}$, the total circumference ratio as $C=\frac{C_{1}}{C_{2}}$, etc. If the total circumference ratio $C$ is equal to the numerosity ratio $N$ then the average diameter ratio $A$ is defined as $A=\frac{C_{1} N_{2}}{N_{1} C_{2}}=\frac{C}{N}=1$. This implies $A \neq N$. nless the two numerosities are equal, i.e., $N=1$ ). Similarly, if the average diameter ratio $A$ is equal to the numerosity ratio $N$, then $C=\frac{A_{1} N_{1}}{A_{2} N_{2}}=N^{2}$, where $C \neq N$. Therefore, the code we present will compare the ratio of one selected continuous magnitude from each group at a time to the numerical ratio. This is in contrast with previous reports that were characterized by attempts to control several variables simultaneously. In addition, we will 


\section{Continuous Magnitudes}

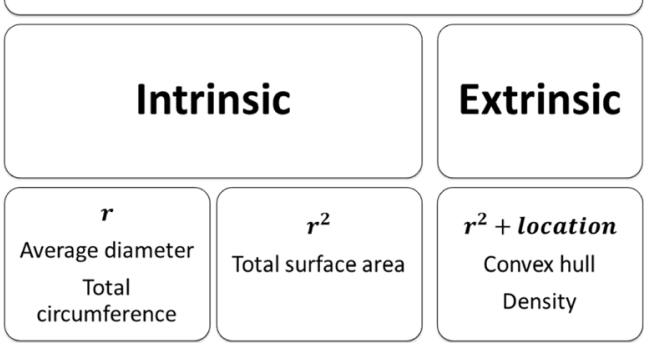

Fig. 2 Division of the continuous magnitudes. Intrinsic magnitudes refer to magnitudes that can be calculated on each individual dot. Extrinsic magnitudes refer to magnitudes that can only be calculated on an array of dots. $r=$ radius

use congruency conditions to disassociate numerosity and the chosen continuous magnitudes (CCM).

In the following section, we will describe the way different continuous ratios are equated to the numerical ratio. We will also demonstrate the utility of our method with a simple nonsymbolic comparison task. The main aim of this demonstration is a proof of concept, showing that equating the ratio of different continuous magnitudes to the numerical ratio would yield differential interference to the numerical and continuous tasks. We chose to conduct this in the subitizing range (i.e., 2-4) as numerosities in this range are considered to be processed automatically and therefore a differential effect in this range would be least expected.

\section{Code algorithm}

The rational underlying this code is to equate the continuous magnitude ratio between two sets of dots to their numerical ratio. We relate to a single continuous magnitude at a time.

$N_{1}, N_{2}$ is the number of dots in each set.

The radii of the dots in the first set will be $r_{1}, r_{2} \ldots$ The radii of the dots in the second set will be $s_{1}, s_{2} \ldots$ We consider the following continuous measures:

Total circumference : $C_{1}=2 \pi \sum_{i=1}^{N_{1}} r_{i}, C_{2}$

$$
=2 \pi \sum_{i=1}^{N_{2}} s_{i}
$$

Average diameter : $A_{1}=\frac{2}{N_{1}} \sum_{i=1}^{N_{1}} r_{i}=\frac{C_{1}}{\pi N_{1}}, A_{2}$

$$
=\frac{C_{2}}{\pi N_{2}}
$$

Total surface area : $S_{1}=\pi \sum_{i=1}^{N_{1}} r_{i}^{2}, \quad S_{2}=\pi \sum_{i=1}^{N_{2}} s_{i}^{2}$
Area of convex hull : $H_{1}, H_{2}$

(custom computation of area according to the specific polygon created by the dots)

Density : $D_{1}=\frac{S_{1}}{H_{1}}, \quad D_{2}=\frac{S_{2}}{H_{2}}$

For the computation in (4), the pixels that make up each dot were first approximated by a polygon with the method circleToPolygon from the package geom2d by David Legland (http://www.mathworks.com/matlabcentral/fileexchange/7844geom2d). Next, the area of the convex hull surrounding all polygons was computed with the Matlab convhull method.

The code randomly assigns radii and locations to the dots in each array according to a user pre-defined range of numerosities and radii (the range of numerosities is limited only by the area on screen). In order to equate the ratio of the CCM to the numerical ratio, the code compresses (shrinks) the array that is larger on the CCM, to the desired ratio. In the congruent condition, the ratio of the $\mathrm{CCM}$ is equal to the numerical ratio:

congruent $=\frac{N_{1}}{N_{2}}=\frac{C C M_{1}}{C C M_{2}}$

In the incongruent condition, the ratio of the $\mathrm{CCM}$ is inverse to the numerical ratio:

incongruent $=\frac{N_{1}}{N_{2}}=\frac{C C M_{2}}{C C M_{1}}$

The congruency of the rest of the continuous magnitudes can be defined by the user. The code documents the ratios between each continuous magnitude in the two to-becompared arrays, to enable statistical monitoring. For convenience, the documented ratios will be calculated by the smaller array (of the magnitude in question) divided by the larger array, so values closer to 1 indicate high similarity (e.g., eight and ten dots will yield the ratio 0.8 , indicating $80 \%$ similarity), and values closer to 0 indicate low similarity (e.g., two and ten dots will yield the ratio 0.2 , indicating $20 \%$ similarity).

For simple notation, we assume in the following that the array denoted by $\mathrm{N}_{2}, C C M_{2}$ is the array with the larger $C C M$, that is, the array that is being rescaled by the compression factor.

The compression for comparing the ratio of average diameter and total surface area was performed in the following manner for the congruent condition:

Congruent Compression Factor $=\frac{N_{2} * C C M_{1}}{N_{1} * C C M_{2}}$ 
For the incongruent condition:

Incongruent Compression Factor $=\frac{N_{1} * C C M_{1}}{N_{2} * C C M_{2}}$

The compression for comparing the ratio of convex hull was performed in the following manner for the congruent condition:

Congruent Compression Factor $=\sqrt{\frac{N_{2} * C C M_{1}}{N_{1} * C C M_{2}}}$

For the incongruent condition:

Incongruent Compression Factor $=\sqrt{\frac{N_{1} * C C M_{1}}{N_{2} * C C M_{2}}}$

If the CCM is an intrinsic magnitude, the radii are subjected to compression. If the CCM is an extrinsic magnitude, in addition to the compression of the radii, the centers of the dots gather closer together by multiplying their coordinates by the compression factor.

\section{Behavioral experiment}

In order to test the effect of equating the ratio of different continuous magnitudes, we created stimuli that were fully congruent or incongruent. In other words, in a congruent stimulus, the ratio of the CCM was equal to the numerical ratio, and the remaining continuous magnitudes were also congruent (but not necessarily in the same ratio). In an incongruent stimulus, the ratio of the CCM was inverse to the numerical ratio, and the remaining continuous magnitudes were incongruent (but not necessarily in the exact inverse ratio). We created three sets of stimuli; in each set a different continuous magnitude was equated - average diameter, total surface area, and convex hull. Since all stimuli were fully congruent or incongruent, the effects found could not be attributed to different levels of congruency (i.e., different number of continuous magnitudes that are congruent with number in different stimuli). Instead, they should be attributed to differences in saliency created from manipulating different continuous magnitudes.

\section{Method}

Participants Seventy-one participants (average age: 24.54 years) completed the experiment. The CCM for 24 participants (eight males) was average diameter, for 21 participants (five males) it was total surface area and for 26 participants (13 males) it was convex hull.
Stimuli The stimuli were dot arrays created with the code. We created three sets of stimuli; in each set a different continuous magnitude was equated - average diameter, total surface area and convex hull. All stimuli were in the subitizing range (i.e., $<5$, excluding 1) so there were 12 types of stimuli for each CCM (i.e., arrays with the following number of dots in the congruent and incongruent conditions): $2-4,4-2,2-3,3-2$, 3-4, 4-3. For examples of the stimuli, see Fig. 3A.

Procedure The procedure was based on that of Leibovich et al. (Leibovich, Henik, \& Salti, 2015). Each trial began with a green fixation point presented on a black screen with a white line bisecting it vertically for $500 \mathrm{~ms}$. The black bisected screen remained in view for $1,000 \mathrm{~ms}$ and then the stimulus appeared for $700 \mathrm{~ms}$. Participants could respond either when viewing the stimuli or up to $1,100 \mathrm{~ms}$ after the stimuli disappeared (see Fig. 3B).

The CCM was manipulated between participants. Namely, one group of participants performed the tasks where the $\mathrm{CCM}$ was average diameter, another group performed the tasks where the CCM was total surface area, and for the third group, the CCM was the convex hull. Each participant preformed two tasks: a numerical task, where participants had to choose (as fast as possible while avoiding errors) the array containing more dots, and a continuous task, where participants had to choose (as fast as possible while avoiding errors) the array containing more white (the dots were white on a black background). The order in which the tasks were administered was counterbalanced. Responses were given by pressing the "q" key for the left array and the "p" key for the right array. Each task began with six practice trials in which participants were given feedback. After the practice block, each task was composed of four blocks of 60 trials each. Altogether, there were 240 trials for each task and 480 trials for the whole experiment, with an equal proportion of congruent and incongruent trials. All participants (in each group) were presented the same set of stimuli. Response times (RTs) and accuracy were recorded.

\section{Results}

Error rates as a dependent measure All analyses were conducted with the program Statistica (version 12). A 3-way analysis of variance (ANOVA) was conducted with CCM (average diameter / total surface area / convex hull) as between subject variables, and task (continuous / numerical) and congruency (congruent / incongruent) as independent within subject variables. CCM affected error rates, $F(2,68)=14.29, p<$ $.001, \eta_{g}^{2}=.31$; when the CCM was convex hull, error rates were significantly lower in comparison to when average diameter or total surface area were the $\mathrm{CCM}, F(1,68)=27.87$, 
A

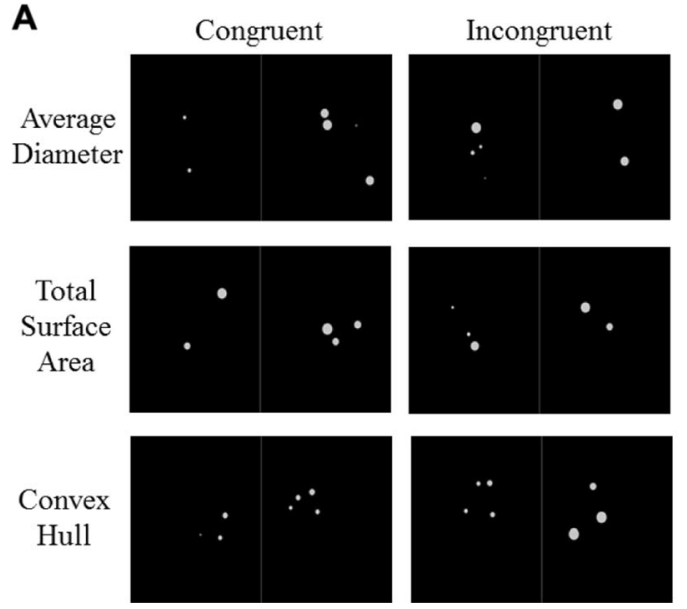

B

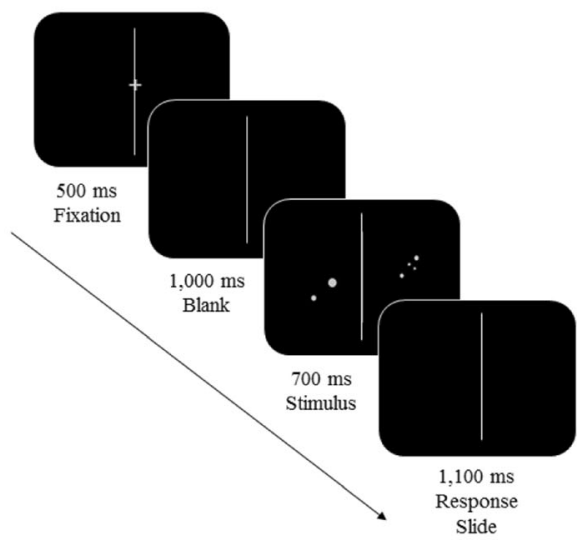

Fig. 3 (A) Examples of congruent and incongruent stimuli for the three chosen continuous magnitudes: average diameter, total surface area and convex hull. (B) An example of a trial in the experiment

$p<.001, \eta_{g}^{2}=.47$. Error rates were similar when average diameter and total surface were the CCMs, $F<1$.

Task also affected error rates, $F(1,68)=110.66, p<.001$, $\eta_{g}^{2}=.37$. Namely, error rates were generally higher for the numerical task than for the continuous task. Congruity also affected error rates, $F(1,68)=103.26, p<.001, \eta_{g}^{2}=.38$. Namely, congruent trials were more accurate than incongruent trials were.

The 3-way interaction between CCM, task and congruency was significant, $F(2,68)=34.36, p<.001, \eta_{g}^{2}=.13$. For the average diameter and total surface area, the congruency effect was bigger in the continuous task, $F(1,68)=132.77, p<.001$, $\eta_{g}^{2}=.23$, and $F(1,68)=8.73, p<.01, \eta_{g}^{2}=.02$, respectively. For the convex hull, the 2-way interaction between task and congruency was not significant, $F<1$.

RT as a dependent measure For the RT analysis, error trials were removed. Table 1 presents the error rates in the different conditions. In addition, trials over or under 3 standard deviations (in RT) for each subject in each condition were removed as well. These trials represented less than $2 \%$ of the trials. Mean RTs of correct trials in each condition for each participant were analyzed with CCM (average diameter / total surface area / convex hull) and order of tasks (continuous-numer$\mathrm{ical} /$ numerical-continuous) as between-subject variables, and task (continuous / numerical) and congruency (congruent / incongruent) as independent within-subject variables in a 4way analysis of variance (ANOVA). We found a main effect for task. Task affected RT, $F(1,67)=17.848, p<.001, \eta_{g}^{2}=$ .04 , namely, the numerical task was faster than the continuous task. Congruity also affected RT, $F(1,67)=104.310, p<$ $.001, \eta_{g}^{2}=.02$. As expected, congruent trials were faster than incongruent trials. We did not find a main effect for CCM, $F$ $(1,67)=2.25, p=.11$, or for order, $F<1$. However, the interaction between task and order was significant so that when the continuous task was first the numerical task was faster, but when the numerical task was first there was no difference, $F(1,67)=6.97, p=.01, \eta_{g}^{2}=.02$. The task and CCM interaction was significant, $F(2,67)=4.58, p=.014, \eta_{g}^{2}$ $=.02$. Further analysis revealed that when the CCM was average diameter or total surface area, the numerical task was faster than the continuous task, $F(1,67)=4.66, p=.03, \eta_{g}^{2}=$ .01 , and $F(1,67)=20.02, p<.001, \eta_{g}^{2}=.05$, respectively. However, when the CCM was convex hull, there was no difference between the tasks, $F<1$ (see Fig. 4). The 3-way interaction between $\mathrm{CCM}$, task and congruency was significant, $F(2,67)=26.3, p<.001, \eta_{g}^{2}=.005$ (see Fig. 4). When the CCM was average diameter, numerosity interfered more than the continuous magnitudes did, that is, the difference in RT between congruent and incongruent trials was bigger in the continuous task than in the numerical task, $F(1,67)=$ $68.12, p<.001, \eta_{g}^{2}=.006$. When the CCM was total surface area or convex hull, the interference was the same, $F<1$ ).

\section{Discussion}

In order to evaluate the benefits of the suggested method, let us start with summarizing the main results of the conducted experiment. First, we found that despite the fact that all sets of stimuli were fully congruent / incongruent, the results differed

Table 1 Error rates in all conditions

\begin{tabular}{llllll}
\hline & \multicolumn{2}{c}{ Continuous Task } & & \multicolumn{2}{c}{ Numerical Task } \\
\cline { 2 - 3 } \cline { 5 - 6 } & Congruent & Incongruent & & Congruent & Incongruent \\
\hline $\begin{array}{l}\text { Chosen continuous } \\
\begin{array}{l}\text { Average } \\
\text { diameter }\end{array}\end{array}$ & 0.02 & 0.36 & & 0.04 & 0.07 \\
$\begin{array}{l}\text { Total surface area } \\
\text { Convex hull }\end{array}$ & 0.12 & 0.25 & & 0.02 & 0.06 \\
\hline
\end{tabular}




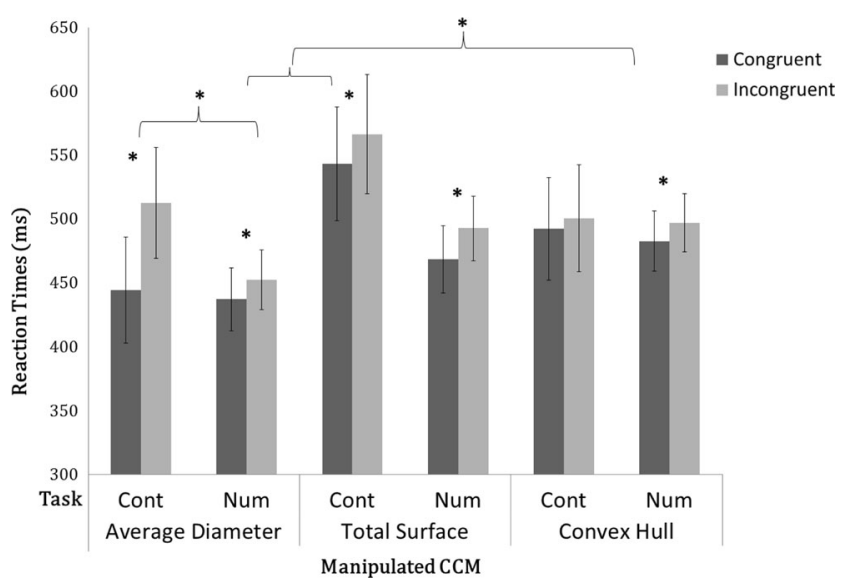

Fig. 4 Response time (RT) results for the three chosen continuous magnitudes (CCM: average diameter, total surface, and convex hull) for each task (continuous and numerical)

according to the CCM. Second, our results suggest that in the subitizing range, a range of numerosities in which numbers are perceived very fast and accurately (Revkin, Piazza, Izard, Cohen, \& Dehaene, 2008; Watson, Maylor, \& Bruce, 2007), comparing the ratio of the convex hull equated the difficulty of the numerical and continuous tasks. When tasks are equally difficult, it is apparent that the interference of numerosity and continuous magnitudes are similar.

The finding that continuous magnitudes interfere in the subitizing range serves as strong evidence for the notion that continuous magnitudes play a role in numerical comparisons. Numerosities in the subitizing range are processed quickly and accurately; accordingly, they are considered automatic (Feigenson et al., 2004). In a recent fMRI (functional magnetic resonance imaging) study, we showed the impact of continuous magnitudes in the subitizing range (Leibovich et al., 2015). Stimuli were produced with the code of Gebuis and Reynvoet (2012b) and subjects performed two tasks: a numerical task and a continuous task (similar to the research herein). On the behavioral level, continuous magnitudes interfered regardless of the task order; however, numerosity interfered only when the numerical task was first. Our results tone down the assertion that continuous magnitudes overpower numerosity. We show that when the numerical and continuous tasks are equally salient, the congruency effects are similar and not affected by context. Our results reveal that saliency, among other things, determines the context. In other words, it is plausible that higher saliency of the continuous properties drove the context effect.

Our experiment examined the effect of equating saliency of numerical and continuous ratios in the subitizing range. We used the subitizing range because it is considered to be a range in which numerosity is processed automatically and thus effects of continuous variables might not be expected. Importantly, we also performed a series of experiments with numerosities ranging from 5-30. In these experiments, we used the code presented in the current article to generate stimuli. The results were similar to those reported here. These experiments show once again the importance of equating different continuous magnitudes, and how they modulate behavior when using different numerosities. We discuss these results and their theoretical implications elsewhere (Katzin, Leibovich, Henik, \& Salti, in preparation).

The main thrust of this paper is to study numerical cognition in a controlled environment. The current method is an evolution of previous and important work aimed at dissociating continuous and numerical magnitudes (Gebuis \& Reynvoet, 2011, 2012b; Halberda, Mazzocco, \& Feigenson, 2008; Piazza et al., 2004). We compared the ratio of continuous magnitudes with the numerical ratio to confront the problem of saliency of one congruency condition over another, and saliency of continuous magnitudes over numerosity (and vice versa).

Recently, DeWind and colleagues (DeWind, Adams, Platt, \& Brannon, 2015) published an insightful paper in which they pointed out the importance of ratio when controlling for continuous magnitudes. They created an estimate of the Weber fraction that was clean of continuous effects and was a better estimate of numerical acuity. To this end, they defined three magnitudes: numerosity, size, and spacing. They provided a code that could calculate these measures from existing arrays. Size and spacing were artificial measures composed mathematically to achieve orthogonality. These artificial measures, although allowing isolation of continuous magnitudes from numerosity as a whole, do not allow characterization of the interactions between specific continuous magnitudes and numerosity. DeWind's method can be applied only on arrays that are homogenous, that is, where all dots are of the same size. The orthogonality of size and spacing would be breached otherwise. Finally, they measured the continuous magnitudes and did not manipulate them. Our method is more accessible in the sense that it operates on the native space and is less restricted. While in the past the intercorrelations of continuous magnitudes were considered an obstacle, we employed them to our benefit. Comparing one continuous magnitude at a time enables examination of the continuous measure on which participants rely on. This enables going from quantitative exploration, namely, how much a continuous magnitude contributes to numerical perception, to asking how does it contribute. To illustrate, if participants rely on density, it insinuates that location of the dots is important for numerical perception and supports the notion of individuation (Dehaene \& Changeux, 1993). On the other hand, if convex hull is the prominent attribute on which participants rely, then individuation is less likely to be a part of the cognitive process of numerical perception.

Other than comparing saliency, our method has several other methodological advantages. Congruency between numerosity and continuous magnitudes is a spectrum that can span from one continuous magnitude up to five congruent continuous magnitudes (or more than the five continuous factors we depicted). The code we provide enables the experimenter to regulate the degree of congruency. In this 
experiment, we used full congruency to increase the odds of there being no subjective correlation and to show that congruency alone is not enough. Nonetheless, full congruency entails an ecological shortfall. For example, density and convex hull are negatively correlated, and by forcing them to be congruent, it creates a situation that is seldom present in real-life situations. These correlations between continuous magnitudes should be considered when creating stimuli.

The code allows flexibility in other domains as well. For example, the code is set to equate the ratio of the CCM to the ratio of the numerosities, but this is not a must. Users can define the ratio they want to use. For example, one could use this code to see the effect of ratio of the CCM on the interference. The ratio can be changed gradually (or otherwise) to create a psychophysical curve and see the impact of continuous magnitudes on numerical processing. In addition, the code currently created stimuli for the comparison task. The user can, with slight changes, alter the code to create stimuli for other tasks like priming and habituation.

In designing this code, we strived to have minimum restrictions and interventions creating the stimuli. The only restriction that is built-in in the code is that we do not allow the dots to touch one another. Other than that, the size and location of the dots are random within a predetermined range. This was done in order to avoid unnecessary correlations.

To conclude, the suggested method and the complimentary code allow generating non-symbolic stimuli for numerical tasks. The method emphasizes the importance of equating the saliency of numerical and continuous magnitudes and opens new venues for future research.

Acknowledgments This work was supported by the European Research Council (ERC) under the European Union's Seventh Framework Programme (FP7/2007-2013)/ERC Grant Agreement 295644 to AH. DK was partially supported by ISF grant no 665/15.

\section{References}

Buckley, P. B., \& Gillman, C. B. (1974). Comparisons of digits and dot patterns. Journal of Experimental Psychology, 103(6), 1131-1136.

Cantlon, J. F., Platt, M. L., \& Brannon, E. M. (2009). Beyond the number domain. Trends in Cognitive Sciences, 13, 83-91. doi:10.1016/j. tics.2008.11.007

Chassy, P., \& Grodd, W. (2012). Comparison of quantities: Core and format-dependent regions as revealed by fMRI. Cerebral Cortex, 22(6), 1420-1430. doi:10.1093/cercor/bhr219

Dehaene, S. (1997). The number sense: How the mind creates mathematics. New York: Oxford University Press.

Dehaene, S., \& Changeux, J. P. (1993). Development of elementary numerical abilities: a neuronal model. Journal of Cognitive Neuroscience, 5(4), 390-407. doi:10.1162/jocn.1993.5.4.390
DeWind, N. K., Adams, G. K., Platt, M. L., \& Brannon, E. M. (2015). Modeling the approximate number system to quantify the contribution of visual stimulus features. Cognition, 142, 247-265. doi:10.1016/j.cognition.2015.05.016

Feigenson, L., Dehaene, S., \& Spelke, E. (2004). Core systems of number. Trends in Cognitive Sciences, 8(7), 307-314. doi:10.1016/j. tics.2004.05.002

Gebuis, T., \& Reynvoet, B. (2011). Generating nonsymbolic number stimuli. Behavior Research Methods, 43(4), 981-986. doi:10.3758 /s13428-011-0097-5

Gebuis, T., \& Reynvoet, B. (2012a). Continuous visual properties explain neural responses to nonsymbolic number. Psychophysiology, 49(11), 1649-1659. doi:10.1111/j.1469-8986.2012.01461.x

Gebuis, T., \& Reynvoet, B. (2012b). The interplay between nonsymbolic number and its continuous visual properties. Journal of Experimental Psychology: General, 141(4), 642-648. doi:10.1037 /a0026218

Gebuis, T., \& Reynvoet, B. (2013). The neural mechanisms underlying passive and active processing of numerosity. NeuroImage, 70, 301307. doi:10.1016/j.neuroimage.2012.12.048

Halberda, J., Mazzocco, M. M. M., \& Feigenson, L. (2008). Individual differences in non-verbal number acuity correlate with maths achievement. Nature, 455, 665-668. doi:10.1038/nature07246

Katzin, N., Leibovich, T., Henik, A., \& Salti, M. (in preparation). Saliency of continuous magnitudes modulates enumeration range.

Leibovich, T., \& Henik, A. (2013). Magnitude processing in nonsymbolic stimuli. Frontiers in Psychology, 4, 375. doi:10.3389 /fpsyg.2013.00375

Leibovich, T., \& Henik, A. (2014). Comparing performance in discrete and continuous comparison tasks. Quarterly Journal of Experimental Psychology, 67(5), 899-917. doi:10.1080/17470218.2013.837940

Leibovich, T., Henik, A., \& Salti, M. (2015). Numerosity processing is context driven even in the subitizing range: An fMRI study. Neuropsychologia, 77, 137-147. doi:10.1016/j. neuropsychologia.2015.08.016

Melara, R. D., \& Algom, D. (2003). Driven by information: A tectonic theory of Stroop effects. Psychological Review, 110(3), 422-471. doi:10.1037/0033-295X.110.3.422

Moyer, R. S., \& Landauer, T. K. (1967). Time required for judgements of numerical inequality. Nature, 215(5109), 1519-1520.

Piazza, M., Izard, V., Pinel, P., Le Bihan, D., \& Dehaene, S. (2004). Tuning curves for approximate numerosity in the human intraparietal sulcus. Neuron, 44(3), 547-555. doi:10.1016/j. neuron.2004.10.014

Revkin, S. K., Piazza, M., Izard, V., Cohen, L., \& Dehaene, S. (2008). Does subitizing reflect numerical estimation? Psychological Science, 19(6), 607-14. doi:10.1111/j.1467-9280.2008.02130.x

Shepard, R. N., Kilpatric, D. W., \& Cunningham, J. P. (1975). The internal representation of numbers. Cognitive Psychology, 7, 82-138. doi:10.1016/0010-0285(75)90006-7

Smets, K., Sasanguie, D., Szücs, D., \& Reynvoet, B. (2015). The effect of different methods to construct non-symbolic stimuli in numerosity estimation and comparison. Journal of Cognitive Psychology, 27(3), 310-325. doi:10.1080/20445911.2014.996568

Watson, D. G., Maylor, E. A., \& Bruce, L. A. M. (2007). The role of eye movements in subitizing and counting. Journal of Experimental Psychology, 33(6), 1389-1399. Retrieved from http://psycnet.apa. orgjournals/xhp/33/6/1389 